\title{
STABLE HOMEOMORPHISMS CAN BE APPROXIMATED BY PIECEWISE LINEAR ONES
}

\author{
BY E. H. CONNELL
}

\section{Communicated by Deane Montgomery, August 23, 1962}

A homeomorphism $h$ of $E_{n}$ or $S_{n}$ onto itself is stable if $\exists$ homeomorphisms $h_{1}, h_{2}, \cdots, h_{m}$ and nonvoid open sets $U_{1}, U_{2}, \cdots, U_{m}$ such that $h=h_{m} h_{m-1} \cdots h_{1}$ and $h_{i} \mid U_{i}=I$ for $i=1,2, \cdots, m$. All orientation preserving homeomorphisms of $E_{n}$ or $S_{n}$ are stable provided $n=1,2$, or 3 . There is no example known in any dimension of an orientation preserving homeomorphism which is not stable. In fact, the conjecture that all orientation preserving homeomorphisms of $E_{n}$ or $S_{n}$ are stable is equivalent to the annulus conjecture (see [3]).

It is known that any homeomorphism of $E_{3}$ onto itself can be approximated by a piecewise linear one (see [2] or [6]). The purpose of this paper is to announce that if $n \geqq 7$ and $h$ is a stable homeomorphism of $E_{n}$ or $S_{n}$ onto itself, then $h$ can be approximated by a piecewise linear homeomorphism, and also, in the case of $E_{n}$, by a diffeomorphism.

The set of all homeomorphisms on $E_{n}$ or $S_{n}$ forms a group under composition and the subset of stable homeomorphisms forms a normal subgroup. The stable group on $S_{n}$ is simple while the stable group on $E_{n}$ is not. Due to this fact, there is a shorter proof in the case of $S_{n}$ than in the case of $E_{n}$, and it is this proof which will be outlined here. The author thanks John Stallings for his assistance.

Notation. $E_{n}$ is Euclidean $n$-space, $S_{n-1}$ is the unit sphere in $E_{n}$, and $O_{n}$ is the open unit ball in $E_{n}$. Thus $O_{n} \cup S_{n-1}=\bar{O}_{n}$. For a given integer $n, O_{n}$ will usually be denoted by $O$. If $U \subset E_{n}$ and $a>0$, $a U=\left\{x \in E_{n}: \exists y \in U\right.$ such that $\left.x=a y\right\} . C(a U)$, the compliment of $a U$, will be denoted by $U a$. Thus, for a given $n, a O$ will be the canonical open ball in $E_{n}$ of radius $a$. If $x, y \in E_{n},|x-y|$ will be the usual distance from $x$ to $y$. If $O$ is the origin and $x \neq O \neq y$, then $\theta\{x, y\}$ will represent the angle in radians between the two line intervals, one joining $O$ to $x$ and the other joining $O$ to $y$. Thus $0 \leqq \theta\{x, y\} \leqq \pi$. A piecewise linear structure (p.w.l. structure) or combinatorial structure on an open subset of $E_{n}$ or $S_{n}$ is a triangulation such that the star of each vertex is a combinatorial cell (see $\$ 3$ of $[10])$. The identity function will be denoted by $I$.

The results of this paper are based primarily on Lemma 1 below, a modification of the Engulfing Lemma (see $\$ 3.4$ of [10]). The proof is omitted. 
Lemma 1. Suppose $E_{n}(n \geqq 4)$ has an arbitrary p.w.l. structure $T, K$ is a finite subcomplex of $T$, $\operatorname{dim} K \leqq n-4, a, b$, and $\epsilon$ are nos. with $0<a<b, \epsilon>0$ and $K \subset b O=b O_{n}$. Then $\exists a$ homeomorphism $h: E_{n} \rightarrow E_{n}$ such that $h$ is p.w.l. relative to $T, h|(a-\epsilon) O=I, h| O b=I, h(a O) \supset K$ and $\theta\{h(x), x\}<\epsilon$ for $x \in E_{n}$.

The proof of Lemma 2 below follows from Lemma 1 and trivial modifications of $\S 4$ of [10] and $\$ 8.1$ of [11]. The proof is omitted.

LEMMA 2. Suppose $E_{n}(n \geqq 7)$ has an arbitrary p.w.l. structure $T$, and $a, b$, and $\epsilon$ are nos. with $0<a<b$ and $\epsilon>0$. Then $\exists a$ homeomorphism $h: E_{n} \rightarrow E_{n}$ such that $h$ is p.w.l. relative to $T, h|(a-\epsilon) O=I, h| O(b+\epsilon)$ $=I, h(a O) \supset b O$ and $\theta\{h(x), x\}<\epsilon$ for $x \in E_{n}$.

Definition. A homeomorphism $h: S_{n} \rightarrow S_{n}$ is said to have property $P$ if for any p.w.l. structure $T$ on $S_{n}$ and any $\epsilon>0$, $\exists$ a homeomorphism $f: S_{n} \rightarrow S_{n}$ such that $f$ is p.w.1. relative to $T$ and $|h(x)-f(x)|<\epsilon$ for $x \in S_{n}$. Let $G_{n}$ be the set of all homeomorphisms on $S_{n}$ which possess property $P$.

OBservation A. $G_{n}$ is a normal subgroup of the group of all homeomorphisms under composition.

Proof. The proof that it is a subgroup is immediate. It will be shown that $G_{n}$ is normal. Suppose $h \in G_{n}$ and $g: S_{n} \rightarrow S_{n}$ is any homeomorphism. Show that $g^{-1} h g \in G_{n}$. Let $T$ and $\epsilon$ be given.

There exists a $\delta>0$ such that if $|x-y|<\delta$, then $\left|g^{-1}(x)-g^{-1}(y)\right|$ $<\epsilon$. Let $T_{1}$ be the p.w.l. structure on $S_{n}$ which is the $g$ image of $T$, $T_{1}=g(T)$. Thus if $v$ is a simplex of $S_{n}$ in the triangulation $T$, then $g(v)$ is a simplex of $S_{n}$ in the triangulation $T_{1}$. Since $h \in G_{n}, \exists$ a homeomorphism $f: S_{n} \rightarrow S_{n}$ which is p.w.l. relative to $T_{1}$ and with $|h(x)-f(x)|$ $<\delta$ for $x \in S_{n}$. Thus $\left|g^{-1} h g(x)-g^{-1} f g(x)\right|<\epsilon$ for $x \in S_{n}$. Note that $g^{-1} f g$ is p.w.l. relative to $T$ because: $g$ is p.w.l. from $T$ to $T_{1}, f$ is p.w.l. from $T_{1}$ to $T_{1}$ and $g^{-1}$ is p.w.l. from $T_{1}$ to $T$. This justifies Observation $\mathrm{A}$.

THEOREM 1. Let $T$ be an arbitrary p.w.l. structure on $S_{n}(n \geqq 7)$ and let $h: S_{n} \rightarrow S_{n}$ be a stable homeomorphism. If $\epsilon>0, \exists a$ homeomorphism $f: S_{n} \rightarrow S_{n}$ such that $f$ is p.w.l. relative to $T$ and $|h(x)-f(x)|<\epsilon$ for $x \in S_{n}$.

Proof. The set of all stable homeomorphisms of $S_{n}$ is a simple, normal subgroup of the group of all homeomorphisms. The fact that it is a normal subgroup is trivial and the fact that it is simple follows from [1] and is even stated explicitly in Theorem 14 of [4]. Therefore, using Observation $\mathrm{A}$, it will follow that $G_{n}$ contains the stable group if $G_{n}$ contains some stable homeomorphism distinct from the 
identity. This will now be shown.

Let $h$ be a symmetric radial expansion, i.e., let $h: E_{n} \rightarrow E_{n}$ be a homeomorphism such that $h(x)=x$ for $\|x\| \geqq 1, h(0)=0, \theta\{h(x), x\}$ $=0$ for all $x$, and if $0<r<1, \exists$ a no. $u(r), r<u(r)<1$ such that $h[r(\bar{O}-O)]=u(r)(\bar{O}-O)$. Let $T$ be any p.w.l. structure on $E_{n}$ and $\epsilon>0$. It will be shown that $\exists f: E_{n} \rightarrow E_{n}$ which is a p.w.l. homeomorphism relative to $T$ and with $f(x)=x$ for $\|x\| \geqq 1$ and $|h(x)-f(x)|<\epsilon$ for $x \in E_{n}$. Since $h$ determines a homeomorphism from $S_{n}$ to itself by defining $h(\infty)=\infty$, this will show that $G_{n}$ is nontrivial and will complete the proof of Theorem 1 .

Let $0=r_{0}<r_{1}<r_{2} \cdots<r_{m+1}=1$ be nos. such that $\left(u\left(r_{i+2}\right)-u\left(r_{i}\right)\right)$ $<\epsilon / 2$ for $i=0,1,2, \cdots,(m-1)$. By Lemma 3, $\exists$ p.w.l. homeomorphisms $f_{1}, f_{2}, \cdots, f_{m}$ such that $f_{i}\left|r_{i-1} O=I, f_{i}\right| O u\left(r_{i+1}\right)=I, \theta\left\{f_{i}(x), x\right\}$ $<\epsilon / 4$ for $x \in E_{n}$, and $f_{i}\left(r_{i} O\right) \supset u\left(r_{i}\right) O$ for $i=1,2, \cdots, m$. Let $f=f_{1} f_{2} \cdots f_{m}$. Now $f$ is a homeomorphism of $E_{n}$ onto $E_{n}$ that is p.w.l. relative to $T$ and $f \mid C(O)=I$. It will be shown that $|f(x)-h(x)|$ $<\epsilon$ for $x \in O$. Let $x \in r_{k+1} O \cap O r_{k}=r_{k+1} O-r_{k} O, 0 \leqq k \leqq m$. Then $f(x)$ $=f_{1} f_{2} \cdots f_{k+1}$ because $f_{t} \mid r_{k+1} O=I$ for $t>k+1$. In fact, $f(x)=f_{k} f_{k+1}(x)$ because $f_{k} f_{k+1}(x) \in O u\left(r_{k}\right)$ and $f_{t} \mid O u\left(r_{k}\right)=I$ for $t<k$. (In the special case $k=0, f(x)=f_{1}(x)$.) Now since $f(x)$ and $h(x) \in u\left(r_{k+2}\right) O \cap O u\left(r_{k}\right)$, $\|f(x)\|$ and $\|h(x)\|$ differ by $<\epsilon / 2$. Since $\theta\{h(x), f(x)\}<\epsilon / 2$ is measured in radians and any radius under consideration is $<1$, it follows that $|h(x)-f(x)|<\epsilon$. This completes the proof.

TheOREM 2. Let $T$ be an arbitrary p.w.l. structure on $E_{n}(n \geqq 7)$. If $h: E_{n} \rightarrow E_{n}$ is a stable homeomorphism and $\epsilon(x): E_{n} \rightarrow(0, \infty)$ is a continuous function, then $\exists$ a homeomorphism $f: E_{n} \rightarrow E_{n}$ such that $f$ is p.w.l. relative to $T$ and $|f(x)-h(x)|<\epsilon(x)$ for $x \in E_{n}$.

Since the stable group on $E_{n}$ is not simple, the trick used in the proof of Theorem 1 cannot be used. A direct construction of $f$ is required. The proof is omitted.

Theorem 3. Suppose $D$ is any $C^{2}$ differentiable structure on $E_{n}(n \geqq 7)$. If $h: E_{n} \rightarrow E_{n}$ is a stable homeomorphism and $\epsilon(x): E_{n} \rightarrow E_{n}$ is a continuous function, then $\exists$ a homeomorphism $f: E_{n} \rightarrow E_{n}$ which is a $C^{2}$ diffeomorphism relative to $D$ and such that $|f(x)-h(x)|<\epsilon(x)$ for $x \in E_{n}$.

Proof. Let $T$ be a $C^{2}$ triangulation of $E_{n}$ which is compatible with $D$ (see [5] or [13]). By Theorem $2, h$ may be approximated by a homeomorphism $f_{1}$ which is p.w.l. relative to $T$. Now by Theorems 5.7 and 6.2 of [7], $f_{1}$ may be approximated by a diffeomorphism $f$. This completes the proof. The theorem remains true if $C^{2}$ is replaced by $C^{\infty}$. 
It is not clear whether or not Theorem 3 remains true when $E_{n}$ is replaced by $S_{n}$. It is known that $E_{n}$ and $S_{n}$ have to be considered as separate cases. For instance, any two differentiable structures on $E_{n}$ are equivalent (except possibly for $n=4$ ) while this is not true on $S_{n}$ (see [10] and [5] respectively).

Let $D_{1}$ and $D_{2}$ be two differentiable structures on $E_{n}(n \geqq 7)$. Let $h: E_{n} \rightarrow E_{n}$ be a diffeomorphism $\bmod D_{1}$. Since diffeomorphisms are always stable, according to Theorem $3, h$ can be approximated by $f$, a diffeomorphism mod $D_{2}$. This type of question might be interesting on $S_{n}$. For instance, let $n=7$ and $D_{1}$ be the ordinary differentiable structure on $S_{7}$ and $D_{2}$ be one of Milnor's bad differentiable structures. Can diffeomorphisms mod $D_{1}$ be approximated by diffeomorphisms mod $D_{2}$ ? They can be approximated by p.w.l. ones by Theorem 1.

If $T_{1}$ and $T_{2}$ are two p.w.1. structures on $E_{n}$ (or $S_{n}$ ), then any homeomorphism p.w.1. relative to $T_{1}$ can be approximated by one p.w.1. relative to $T_{2}$. This follows from Theorem 2 (resp. Theorem 1) and the fact that p.w.1. homeomorphisms are stable.

\section{REFERENCES}

1. R. D. Anderson, The algebraic simplicity of certain groups of homeomorphisms, Amer. J. Math. 80 (1958), 955-963.

2. R. H. Bing, An alternate proof that 3-manifolds can be triangulated, Ann. of Math. (2) 69 (1959), 37-65.

3. M. Brown and H. Gluck, Stable structures on manifolds. I. Homeomorphisms of $S_{n}$, Ann. of Math. (to appear).

4. G. M. Fisher, On the group of all homeomorphisms of a manifold, Trans. Amer. Math. Soc. 97 (1960), 193-212.

5. J. Milnor, On the relationship between differentiable manifolds and combinatorial manifolds, mimeographed notes, Princeton University, Princeton, N. J., 1956.

6. E. E. Moise, Affine structures in 3-manifolds. IV. Piecewise linear approximations of homeomorphisms, Ann. of Math. (2) 55 (1952), 215-222.

7. J. Munkres, Obstructions to the smoothing of piecewise-differentiable homeomorphisms, Ann. of Math. (2) 72 (1960), 521-554.

8. R. Penrose, J. H. C. Whitehead and E. C. Zeeman, Imbedding of manifolds in Euclidean space, Ann. of Math. (2) 73 (1961), 613-623.

9. J. Stallings, Polyhedral homotopy spheres, Bull. Amer. Math. Soc. 66 (1960), $485-488$.

10. - The piecewise-linear structure of euclidean space, Proc. Cambridge Philos. Soc. 58 (1962), 481-488.

11. - On topologically unknotted spheres, Ann. of Math. (to appear).

12. J. H. C. Whitehead, Simplicial spaces, nuclei and m-groups, Proc. London Math. Soc. 45 (1939), 243-327.

13. — On $C^{1}$-complexes, Ann. of Math. (2) 41 (1940), 809-824.

14. M. K. Fort, Topology of 3-manifolds, Prentice-Hall, Englewood Cliffs, N. J., 1962, pp. 198-204.

INSTITUTE FOR ADVANCED STUDy 